\title{
Calcium channel blockers reduce the antiplatelet effect of clopidogrel
}

\author{
Jolanta M Siller-Matula*1, Irene Lang ${ }^{2}$, Günter Christ ${ }^{2}$ and Bernd Jilma ${ }^{1}$
}

\author{
Address: ${ }^{1}$ Department of Clinical Pharmacology, Medical University of Vienna, 1090 Vienna, Austria and ${ }^{2}$ Department of Cardiology, Medical \\ University of Vienna, 1090 Vienna, Austria \\ Email: Jolanta M Siller-Matula* - jolanta.siller-matula@meduniwien.ac.at \\ * Corresponding author
}

from $14^{\text {th }}$ Scientific Symposium of the Austrian Pharmacological Society (APHAR)

Innsbruck, Austria. 2I-22 November 2008

Published: 5 November 2008

BMC Pharmacology 2008, 8(Suppl I):A47 doi:I0.1186/I47I-2210-8-SI-A47

This abstract is available from: http://www.biomedcentral.com/I47I-22I0/8/SI/A47

(c) 2008 Siller-Matula et al; licensee BioMed Central Ltd.

\section{Background}

Clopidogrel is activated by CYP3A4 which also metabolizes calcium channel blockers of the dihydropyridine class. Due to the known CYP3A4 inhibition by calcium channel blockers, we hypothesized that there might be a drug-drug interaction between clopidogrel and dihydropyridines in patients with coronary artery disease.

\section{Materials and methods}

Responsiveness to clopidogrel was assessed by the vasodilator stimulated phosphoprotein (VASP) phosphorylation assay and aggregometry in 200 patients with coronary artery disease undergoing percutaneous coronary intervention.

\section{Results}

The platelet reactivity index (PRI in the VASP assay, normal range 69-100\%) was higher in patients on both clopidogrel and calcium channel blockers (61\%) as compared to patients on clopidogrel without calcium channel blockers (48\%). The absolute difference was $13 \%$ (95\%CI: $6-20 \% ; \mathrm{p}=0.001)$ and the relative difference approached $21 \%$. A reduced effect of clopidogrel (PRI $>69 \%$ ) was seen in $40 \%$ of patients with concomitant calcium channel blocker treatment compared to $20 \%$ without ( $\chi^{2}$-test: $\left.p=0.008\right)$. Intake of calcium channel blocker remained an independent predictor of reduced platelet inhibition by clopidogrel after adjustment for cardiovascular risk factors. This corresponded to an increased platelet aggregation of similar magnitude $(\mathrm{p}<0.05)$ and was associated with adverse clinical outcome. In vitro incubation with calcium channel blockers (nimodipine, verapamil, amlodipine and diltiazem) did not alter the PRI or the ADP-induced platelet aggregation of patients on clopidogrel. This indicates that the negative effect occurs in vivo, conceivably at the level of the CYP3A4 cytochrome.

\section{Conclusion}

Co-administration of calcium channel blockers is associated with a decreased platelet inhibition by clopidogrel. 